\title{
Cystic renal diseases: role of ultrasound. Part II, genetic cystic renal diseases
}

\author{
Adnan Kabaalioglu', Nesrin Gunduz ${ }^{2}$, Ayse Keven ${ }^{3}$, Emel Durmaz ${ }^{3}$, Mine Aslan ${ }^{4}$, Ahmet Aslan ${ }^{4}$, \\ Serkan Guneyli ${ }^{1}$
}

${ }^{1}$ Department of Radiology, Koc University School of Medicine, Istanbul, Turkey, ${ }^{2}$ Department of Radiology, Istanbul Medeniyet University, School of Medicine, Istanbul, Turkey, ${ }^{3}$ Department of Radiology, Akdeniz University School of Medicine, Antalya, Turkey, ${ }^{4}$ Department of Radiology, King Hamad University Hospital, Muharraq, Bahrain

\begin{abstract}
Kidney cysts are quite common in adults. Though small simple renal cysts in an adult over 30-40 years of age are not too unusual, however, if the same cysts are seen in a child, and especially if there are additional findings, then several diagnostic possibilities may come to mind. The role of ultrasound, together with the help of intravenous contrast agents and Doppler mode, are very critical in describing the morphologic features and follow-up of the complex or multiple and bilateral renal cysts. These sonographic signs are occasionally specific for diagnosis, but in many cases sonographic clues should be evaluated together with the other genetic and clinical data to reach diagnosis.

The first part of this pictorial essay included the introduction into the subject and the classification of non-genetic cystic renal diseases. The key features for the non-genetic cystic renal diseases are illustrated. In the second part, eye-catching features of genetic cystic renal diseases are demonstrated.
\end{abstract}

Keywords: cyst; cystic; Doppler; kidney; ultrasound (US)

\section{Introduction}

In this second part, eye-catching features of genetic cystic renal diseases are demonstrated. In general, cysts are probably the most frequent abnormal findings to be reported during sonography due to their high prevalences. Actually, simple cysts, even if they are multiple, are considered to be almost a normal developmental variation in the elderly. On the other hand, even a tiny simple cyst in a child may be the strong evidence of a genetic cystic renal disease.

Received 26.07.2020 Accepted 29.09.2020

Med Ultrason

2022, Vol. 24, No 1, 107-113

Corresponding author: Assoc. Prof. Dr. Serkan Guneyli, M.D.

Department of Radiology, Koc University School of Medicine, Istanbul, Turkey

Phone: 00905336981477

Fax: 00902123113410

E-mail: drserkanguneyli@gmail.com
Genetic cystic renal diseases include autosomal dominant polycystic kidney disease (ADPKD), autosomal recessive polycystic kidney disease (ARPKD), juvenile nephronophthisis / medullary cystic kidney disease complex, and multiorgan syndromes with pluricystic kidneys.

\section{Genetic cystic renal diseases}

\section{Autosomal dominant polycystic kidney disease}

ADPKD is the most well-known and most frequent hereditary cystic disease which usually manifests in the third decade of life. However, some patients are incidentally discovered in their sixth or seventh decades; the genetic basis for these almost $15 \%$ of cases, have been discovered in recent years. Chromosome 4 is responsible in these mild cases instead of chromosome 16 . In the elderly, multiple bilateral cysts due to aging, may sometimes create a suspicion of ADPKD, then kidney volume and other clues may be required for a certain diagnosis. 
Diagnosis with ultrasound (US) is easy in overt cases, with bilateral large kidneys, full of multiple cysts that compress and hide the parenchyma, with a variable function (fig 1). Liver and other organ cysts may accompany the disease. Diagnosis may be difficult in children or young adults when only one or few tiny cysts can be seen and the kidney volume is normal or borderline (fig 2).

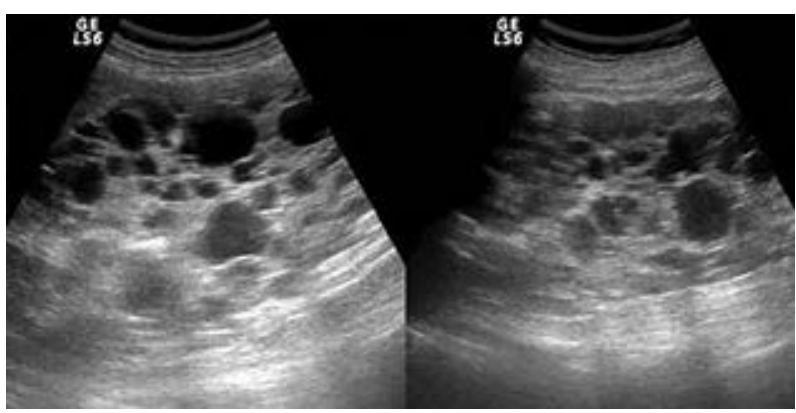

Fig 1. Typical ADPKD; bilateral enlarged kidneys with hundreds of tiny cysts compressing the parenchyma.

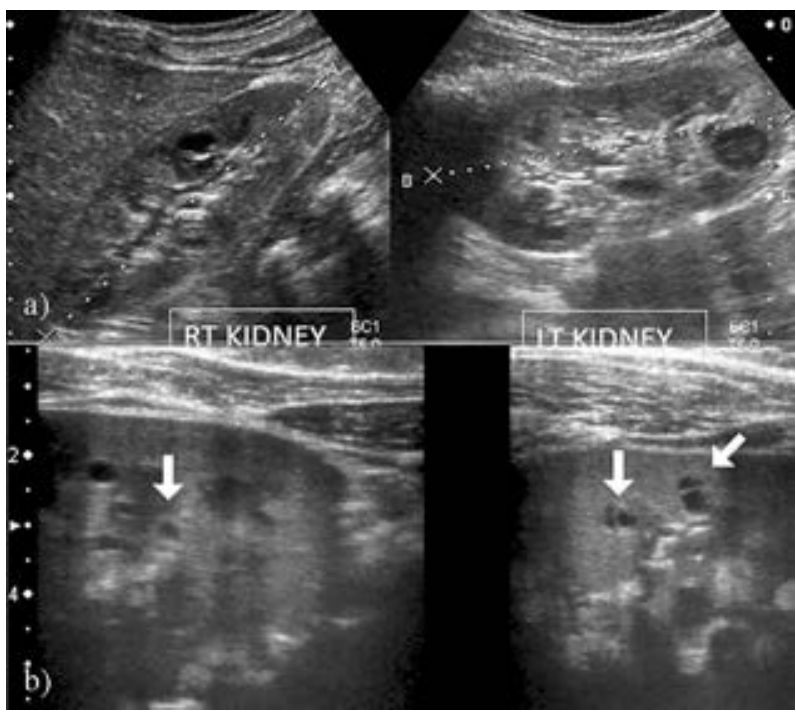

Fig 2. a) 11-year-old boy. Only 2 cysts are seen by the convex probe. b) Linear probe shows more than 5 tiny cysts (arrows) in each kidney of the same patient with ADPKD, similar to the findings of his mother.

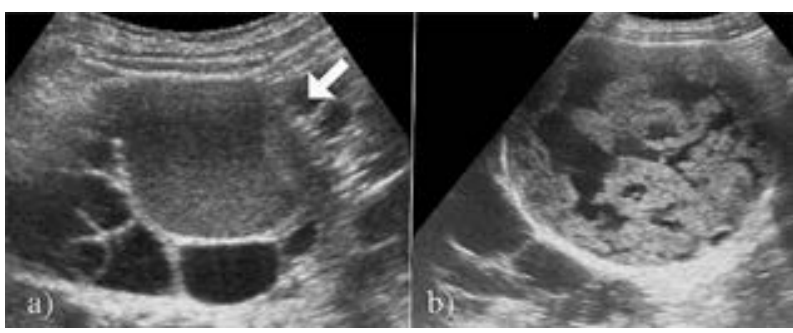

Fig 3. a) A complex hemorrhagic cyst (arrow) in an adult with ADPKD. b) A complex hemorrhagic cyst in another adult with ADPKD.

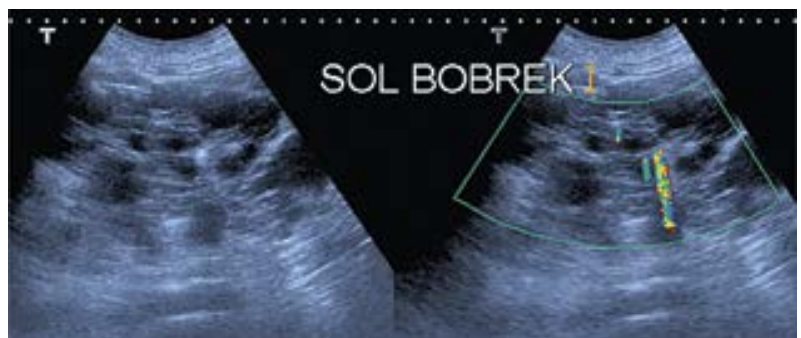

Fig 4. Impact of twinkling artifact in color Doppler US mode, to ease the diagnosis of nephrolithiasis in this 45-year-old male with ADPKD.

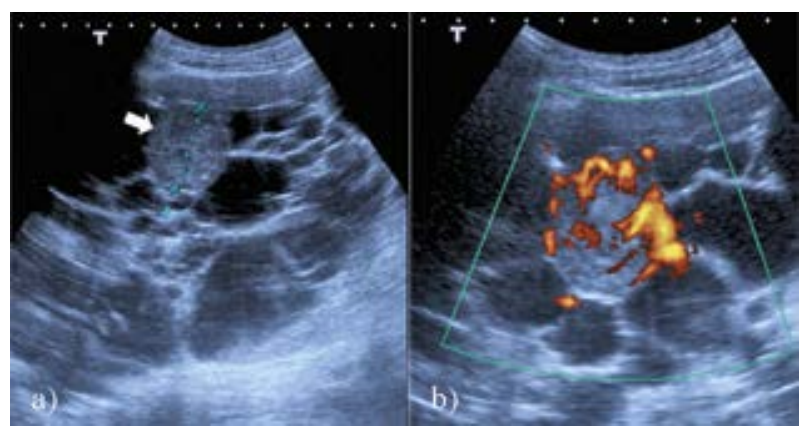

Fig 5. a) A solid mass (arrow) in a 55-year-old male with ADPKD which was found to be a RCC after resection. b) Power Doppler US shows the vascularity of the mass.

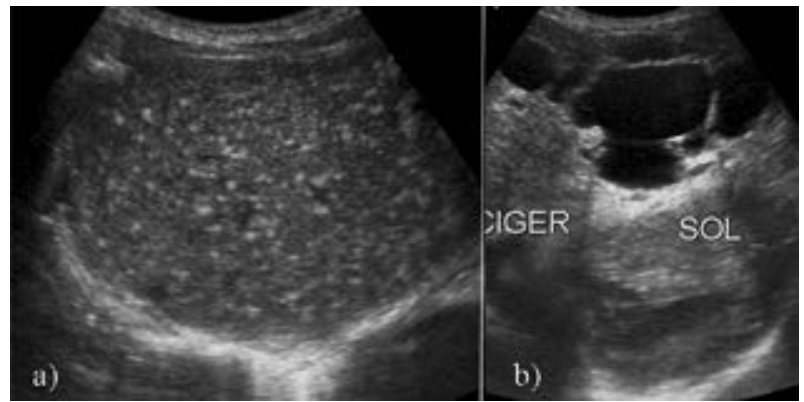

Fig 6. a) Multiple tiny echogenic foci in the liver of a 70-yearold male with ADPKD. The lesions had low density on CT (not shown). b) Multiple cysts were also present in the liver.

The high-resolution linear probes can certainly change the diagnosis in the majority of children screened by US because of a parent with known ADPKD.

Hemorrhage and infection rates of cysts in ADPKD are interestingly higher than the simple usual cysts in the normal population [1] (fig 3). Calcifications and stones are also frequent and may be difficult to find out by US alone in a big mass; Doppler US and CT may help (fig 4). Renal cell carcinoma (RCC) incidence is not increased in patients with ADPKD; but if they start having dialysis, then the risk increases (fig 5). Rarely, biliary hamartomas may be seen; cysts in ADPKD and biliary hamartomas are thought to develop from the interrupted remodeling 


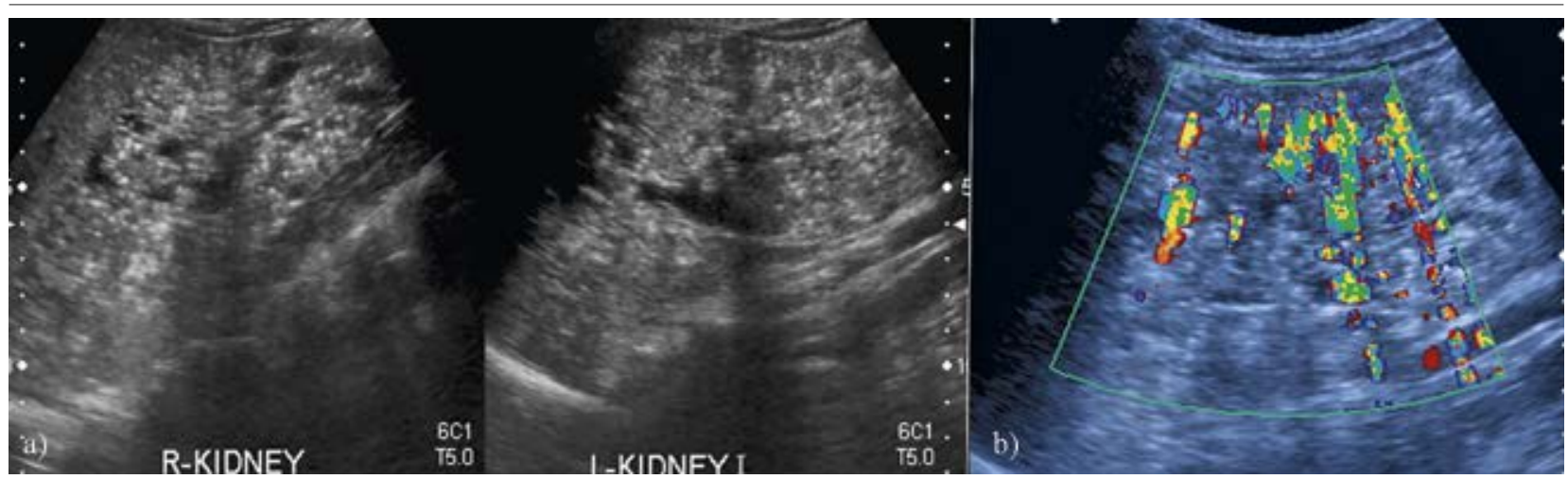

Fig 7. a) Bilateral big and echogenic kidneys in a 6-year-old boy. b) Intense twinkling artifacts are seen.

of the ductal plates during the late phase of embryologic development [1] (fig 6).

Autosomal recessive polycystic kidney disease

ARPKD is primarily a pediatric disease that is more severe than ADPKD; almost half of the patients die either in utero or soon after birth. The liver is always involved with progression to end-stage disease [2]. The kidneys get larger, starting prenatally, and individual cysts may not be seen especially in the early phase; instead echogenic parenchyma is noted. The echogenic medullary region may be described as "nephrocalcinosis" and almost all cases show strong twinkling artifacts (fig 7, fig 8). The tiny cysts or rather dilated tubules can be seen with highresolution probes as fusiform structures (fig 9a). Another frequent sonographic observation is the lateralization of the gallbladder due to relative atrophy of the right liver lobe and hypertrophy of the left liver lobe (fig 9b). Besides diffuse liver parenchymal heterogeneity, biliary dilatations and biliary cysts can be diagnosed by US as Caroli disease accompanying ARPKD (fig 10).

Differential diagnosis includes other genetic syndromes which may have a similar kidney appearance with extrarenal findings and unique genetic features [3].

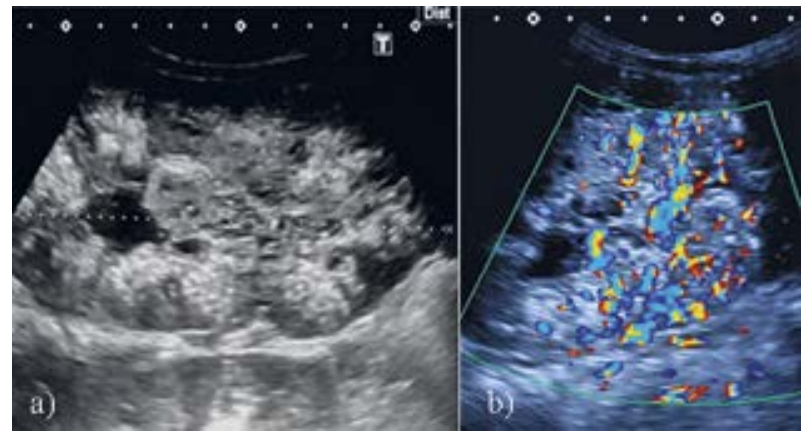

Fig 8. a) Typical, big for age (130 mm) echogenic kidneys with tiny cysts and dilated tubules in a 7-month-old girl with ARPKD. b) Striking parenchymal twinkling artifacts occur by Doppler US mode.

\section{Nephronophthisis / Medullary cystic kidney disease complex}

This group of rare genetic diseases with already some controversies in classifications, have recently been split as "nephronophthisis" and "autosomal dominant tubulointerstitial kidney disease" (ADTKD) in a consensus statement of an international working group composed mainly of pediatric nephrologists and pediatric radiolo-

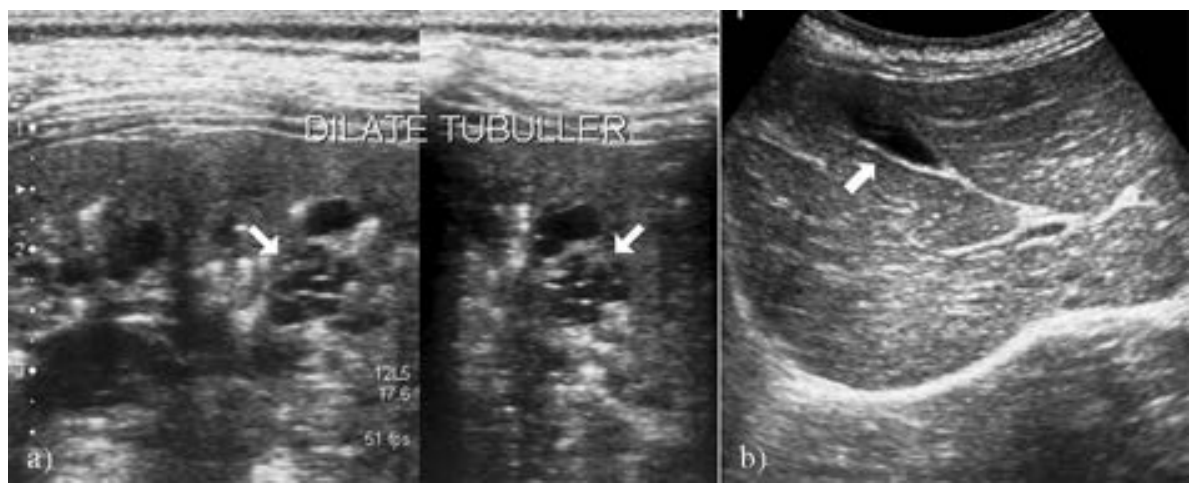

Fig 9. a) Dilated fusiform tubules (arrows) and tiny cysts in a 4-year-old boy with ARPKD. b) Heterogeneity in the liver and lateralization of the gallbladder (arrow). 


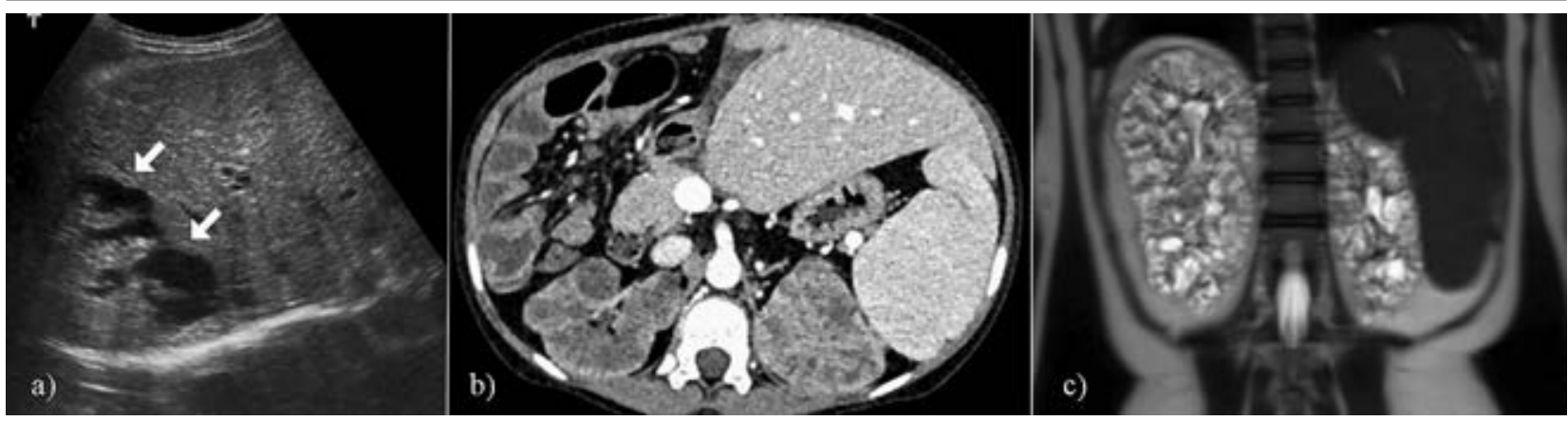

Fig 10. a) Irregularly dilated bile ducts (arrows) in a 9-year-old girl with ARPKD and Caroli syndrome. Axial CT (b) and coronal MRI (c) of the same patient after liver transplantation showing the polycystic kidneys, transplanted left liver lobe, and an enlarged spleen.

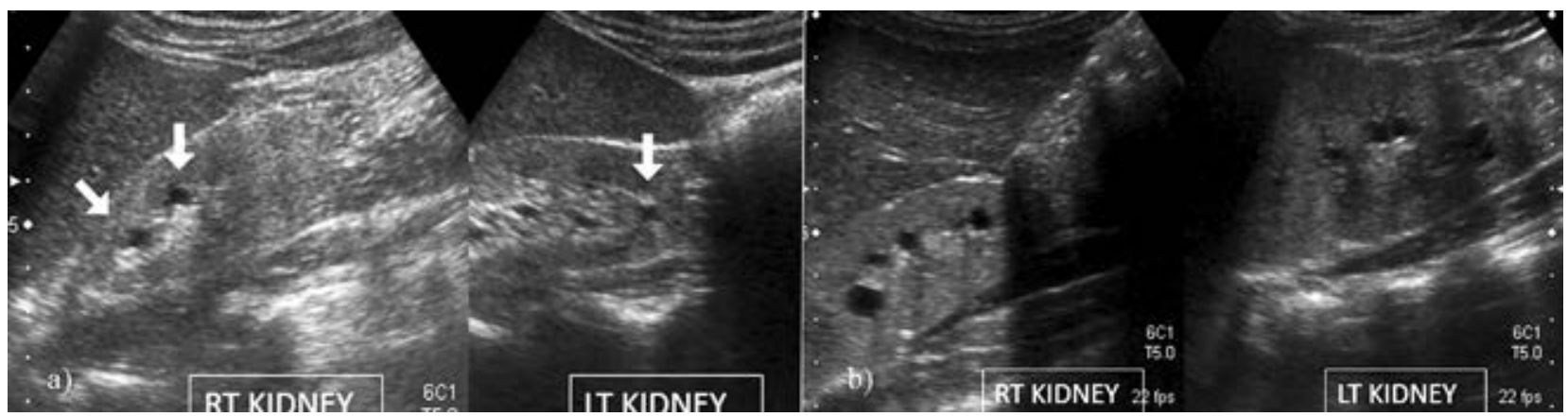

Fig 11. Two different cases followed up with a probable diagnosis of nephronophthisis. Bilateral echogenic small kidneys with tiny medullary cysts (arrows) are shown in a 19-year-old (a) and a 10-year-old girl (b).

gists [3]. ADTKD includes the previous "uremic medullary cystic kidney diseases" and some more genetic cystic diseases. US or other imaging methods are not so useful and specific in these diseases; the kidneys may be normal or small in size, the parenchyma may be echogenic, and medullary cysts may not always be seen [3]. The most important clue is the specific location of small cysts (almost always less than $2 \mathrm{~cm}$ ), in the cortico-medullary junction (fig 11). Caroli disease and hepatic fibrosis may accompany and should be checked. Genetic analysis is mandatory for verification of diagnosis.

\section{Multiorgan syndromes with pluricystic kidneys}

In addition to ADPKD and ARPKD, cystic kidney disease is a common feature of ciliopathies with extrarenal manifestations which require careful clinical workup to identify the underlying genetic disorder, especially in children. In the recent years, our understanding of the basis of polycystic kidney disease has increased substantially and more than 100 genes have been discovered to be involved in these cystic kidney diseases with enormous complexity $[2,4]$.

The most common disease in this diverse group is tuberous sclerosis complex (TSC). This disorder is usually identified in infants and children based on characteristic skin lesions, seizures, and cellular overgrowth or hamar- tomas in the heart, brain, and kidneys [5]. Although renal involvement is asymptomatic initially, more than $75 \%$ die of renal failure. The disease can be suspected in utero by the presence of cardiac rhabdomyomas (fig 12). The most frequent renal finding is angiomyolipoma (AML) with an incidence of $40-90 \%$ (fig 13). Fat-poor AMLs

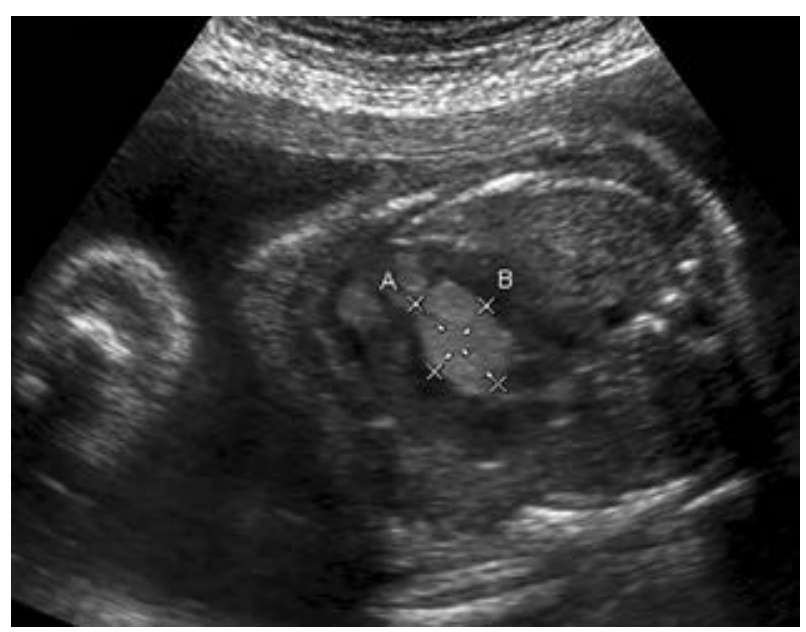

Fig 12. The prenatal US shows a cardiac echogenic mass reported as probable rhabdomyoma. The child is still on followup with TSC diagnosis. 

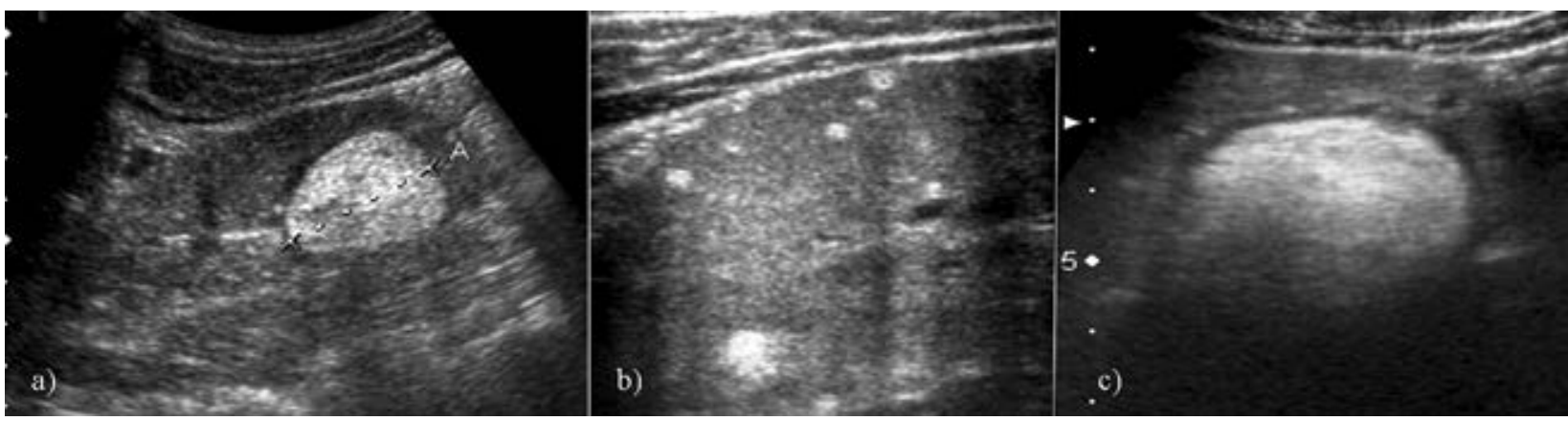

Fig 13. a-c) Multiple, bilateral, different-sized, and highly echogenic angiomyolipomas in an 18-year-old boy with TSC.

are not rare and they can be confusing, since RCCs may also be rarely seen in TSC patients. In these patients, US may not be sufficient for the diagnosis, and CT or magnetic resonance imaging (MRI) is required for the differential diagnosis. Bilateral multiple cysts are seen in $15-40 \%$ of patients (fig 14). The cysts and the kidneys are bigger earlier in childhood in the TSC2/PKD1 CGS (Tuberous Sclerosis Complex 2/Polycystic Kidney Disease 1 Contagious Gene Syndrome) form of the disease [6] (fig 15). AMLs larger than 3-4 cm should be observed and evaluated for the need of embolisation against the bleeding risk (fig 16).

Von Hippel-Lindau disease, which is a rare disorder is characterized by cysts, cystic and hypervascular visceral neoplasms. Renal lesions, including renal cysts and RCC are seen in $30-75 \%$ of cases [7]. Pancreatic cysts and cystadenomas, neuroendocrine tumors and pheochromocytomas may be also seen (fig 17).

In Meckel-Gruber syndrome, most cases are prenatally detected $(90 \%)$ and die in utero or soon after birth [8]. Although many multiorgan anomalies are seen, the triad of polycystic kidneys, encephalocele, and polydactyly is the most common finding [8] (fig 18).

The diagnosis of HNF1B-associated disease can not be made with imaging alone and requires genetic confirmation [3]. It is considered to belong to the ADTKD

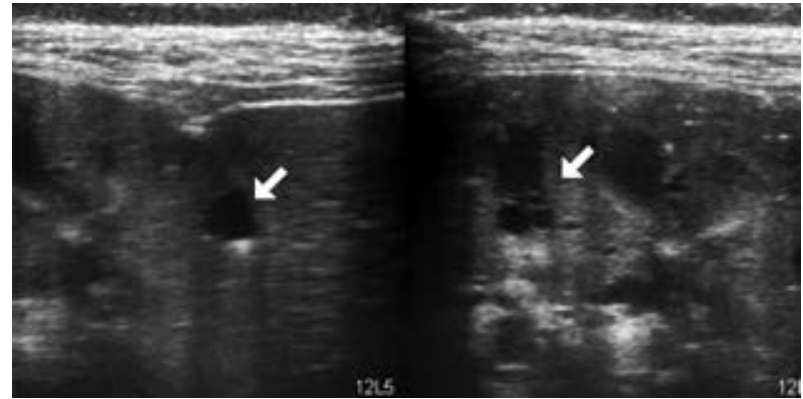

Fig 14. Multiple, bilateral, and tiny cysts (arrows) in a 9-yearold boy with TSC.

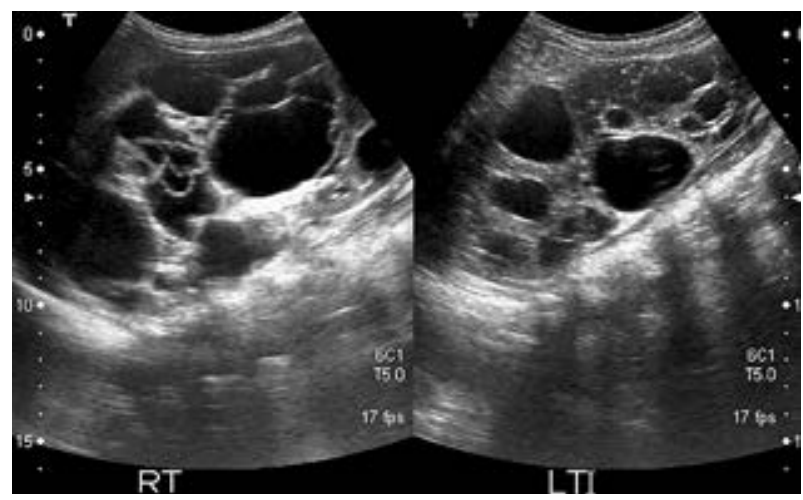

Fig 15. Multiple big cysts in big kidneys $(16 \mathrm{~cm})$ of a 4-yearold boy with Type 2 TSC.

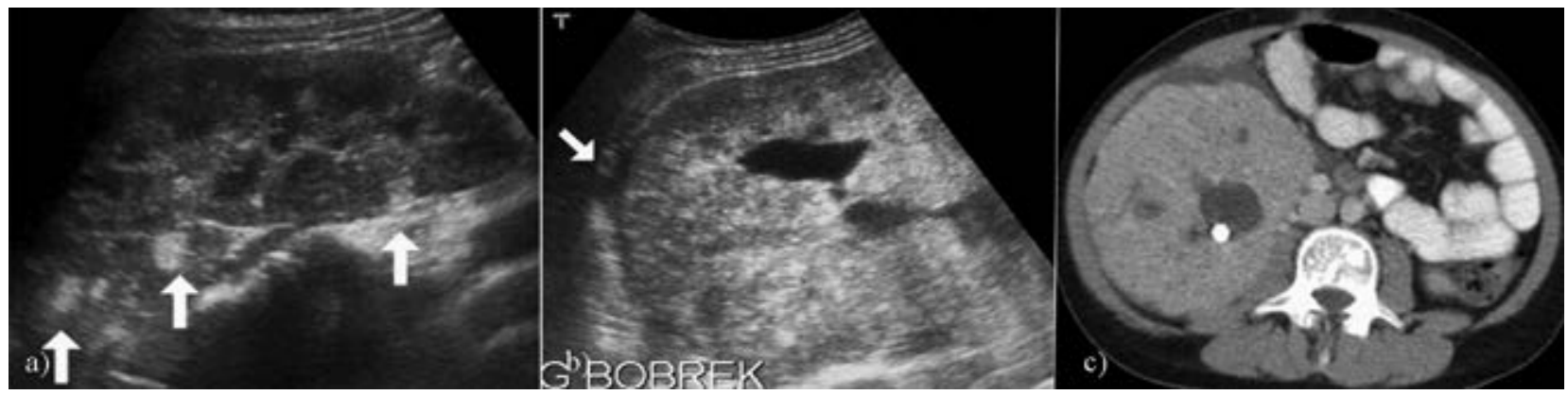

Fig 16. a) Multiple AMLs (arrows) in the right kidney of a 17-year-old boy with TSC. One of them was a huge (14 cm in diameter) fat-poor AML and embolised. This lesion (arrow) is shown on US (b) and CT (c). 


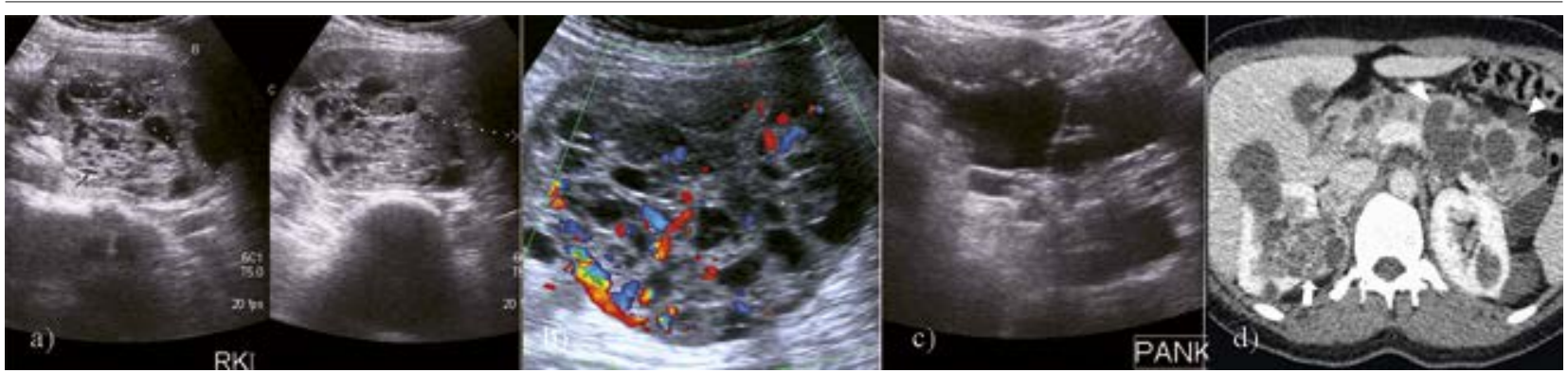

Fig 17. A 28-year-old female with von Hippel-Lindau disease: a) Complex right renal cystic mass of $8 \mathrm{~cm}$; b) Doppler US revealing hypervascularity of cystic RCC which was bilateral; c) Pancreatic cysts are shown on US; d) CT shows both the cystic RCC (arrow) in the right kidney and multiple pancreatic cysts (arrowheads).

group and is the most common cause of hyperechoic kidneys on the prenatal US. However, usually the renal US findings are nonspecific; may mimic ARPKD, may be unilateral, may reflect dysplasia, and may be normal. Associated anomalies, especially female genital anomalies should be screened with US [3].

McKusick-Kaufman syndrome may have similar findings with HNF1B disease, and polydactyly may accompany genital anomalies and renal cysts (fig 19).

Bardet-Biedl syndrome, Beckwith-Wiedemann syndrome, glomerulocystic kidney disease, Zellweger syndrome, Joubert syndrome, Oral-facial-digital syndrome, and Jeune syndrome are relatively well-known hereditary multiorgan syndromes with cystic renal changes. However, the whole family is very large and includes multiple syndromes. Without genetic testing, diagnosis and differential diagnosis is not easy. US or MRI is most often not specific and not helpful in the initial diagnostic phase, and sonography is almost always used during follow-up.

\section{Conclusion}

Cystic renal diseases are a large spectrum containing many genetic and non-genetic conditions, in which sonographic signs might be very helpful in diagnosis if the meaning, sensitivity, and specificity of these US signs

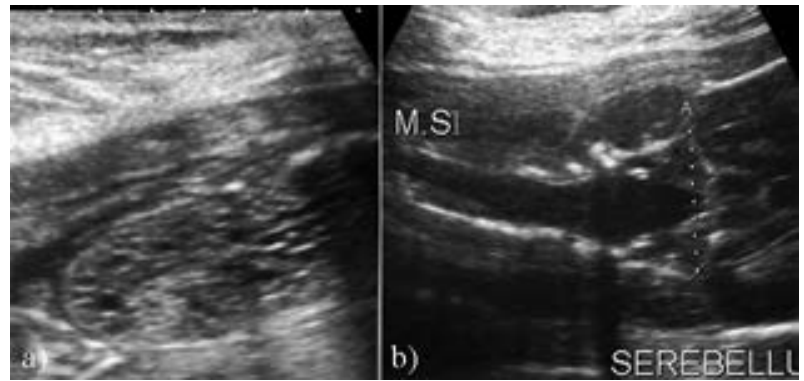

Fig 18. a) Big and polycystic kidneys and b) encephalocele in a fetus with anhydramnios.

are known. Doppler and CEUS features are especially important for differential diagnosis of neoplastic cystic renal masses from other non-neoplastic cysts.

Acknowledgement: The authors thank Gokce Akgunduz Annac for creative illustrations in fig 1 in the first part.

\section{Conflict of interest: None.}

\section{References}

1. Kabaalioglu A, MacLennan GT. Cystic Diseases of the Kidney. In: Dogra VS, MacLennan GT (eds.). Genitouri-

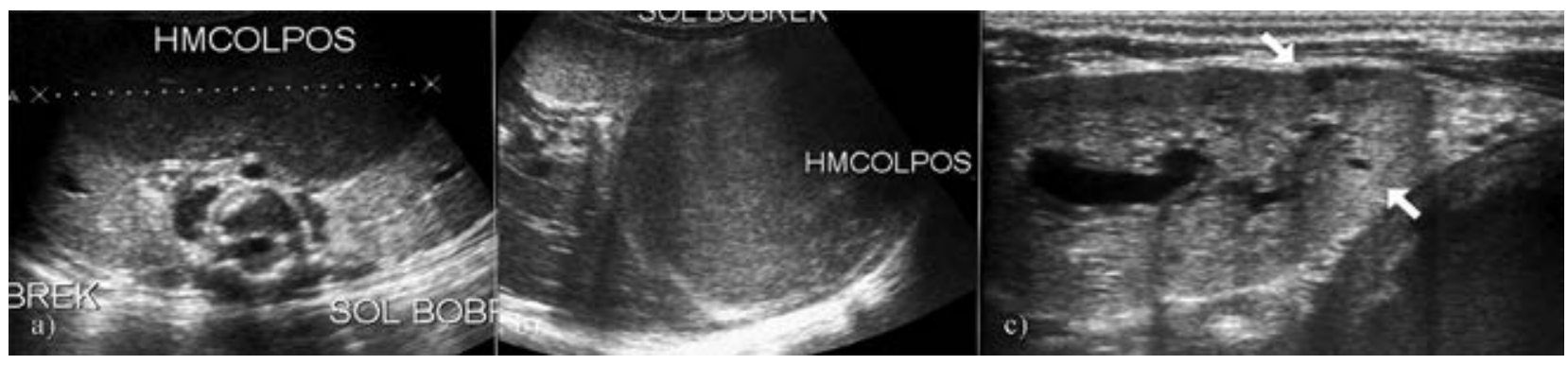

Fig 19. Abdominal mass measuring $8 \mathrm{~cm}$ in a 10-month-old girl with polydactyly of both hands and feet was diagnosed as hydrometrocolpos due to vaginal atresia. Transverse (a) and longitudinal (b) sonograms show the huge abdominal midline cystic mass with internal echoes. c) Both kidneys are enlarged and appear polycystic (arrows). 
nary Radiology: Kidney, Bladder and Urethra. SpringerVerlag London, 2013:95-119.

2. Bergmann C. Genetics of Autosomal Recessive Polycystic Kidney Disease and Its Differential Diagnoses. Front Pediatr 2018;5:221

3. Gimpel C, Avni EF, Breysem L, et al. Imaging of Kidney Cysts and Cystic Kidney Diseases in Children: An International Working Group Consensus Statement. Radiology 2019;290:769-782.

4. Müller RU, Benzing T. Cystic Kidney Diseases From the Adult Nephrologist's Point of View. Front Pediatr 2018;6:65.

5. Randle SC. Tuberous Sclerosis Complex: A Review. Pediatr Ann 2017;46:e166-e171.
6. Back SJ, Andronikou S, Kilborn T, Kaplan BS, Darge $\mathrm{K}$. Imaging features of tuberous sclerosis complex with autosomal-dominant polycystic kidney disease: a contiguous gene syndrome. Pediatr Radiol 2015;45:386395.

7. Katabathina VS, Kota G, Dasyam AK, Shanbhogue AK, Prasad SR. Adult renal cystic disease: a genetic, biological, and developmental primer. Radiographics 2010;30:15091523.

8. Barisic I, Boban L, Loane M, et al. Meckel-Gruber Syndrome: a population-based study on prevalence, prenatal diagnosis, clinical features, and survival in Europe. Eur J Hum Genet 2015;23:746-752. 